\title{
The role of empirical albendazole treatment in idiopathic hypereosinophilia - a case series
}

\author{
Eleonora Vaisben $M D^{1}$, Ronen Brand $M D^{2}$, Anas Kadakh $M D^{1}$, Faris Nassar $M D^{1,3}$
}

\begin{abstract}
E Vaisben, R Brand, A Kadakh, F Nassar. The role of empirical albendazole treatment in idiopathic hypereosinophilia - a case series. Can J Infect Dis Med Microbiol 2015;26(6):323-324.

Hypereosinophilia is usually defined as a blood eosinophil count $>1500 / \mu \mathrm{L}$. A broad variety of conditions are associated with hypereosinophilia. The present report describes three cases of hypereosinophilia, in which a thorough history, physical examination, laboratory and imaging investigations were unable to detect any abnormalities. Albendazole was empirically administered in all three cases, with complete normalization of eosinophil counts thereafter. Empirical treatment with albendazole for patients presenting with hypereosinophilia should be strongly considered.
\end{abstract}

Key Words: Albendazole; Hypereosinophilia; Hypereosinophilic syndrome levated eosinophil count is not a rare finding in clinical practice. An eosinophil count $>500 / \mu \mathrm{L}$ is considered to be mildly elevated; however, hypereosinophilia is usually defined as a blood eosinophil count $>1500 / \mu \mathrm{L}$. Severely elevated eosinophil count $(>5000 / \mu \mathrm{L})$ is usually, but not uniformly, associated with eosinophilic infiltration of tissues, which can lead to life-threatening organ damage. A broad variety of allergic, infectious, neoplastic and idiopathic diseases are associated with hypereosinophilia, and should all be taken into consideration during the evaluation of a patient presenting with hypereosinophilia.

A practical approach to the diagnosis and management of hypereosinophilia has been discussed in the literature; however, no current guidelines have been established. Failure to detect any underlying cause of eosinophilia or evidence of hypereosinophilic syndrome (HES) following a thorough evaluation can cause frustration for the patient as well the treating physician.

The present report describes three cases of severe hypereosinophilia, hospitalized for evaluation in our internal medicine department from 2011 to 2012. In all three cases, no apparent cause of hypereosinophilia could be found, and an HES diagnosis could not be established. A novel approach of empirical treatment with albendazole was initiated with complete resolution of hypereosinophilia in all three cases. The role of empirical albendazole treatment is also discussed.

\section{CASE 1}

A 46-year-old man was admitted to the internal medicine department for evaluation of an incidental finding of leukocytosis and hypereosinophilia. He complained of a pruritic skin rash on his lower extremities for the past eight months. He denied any fever, weight loss or loss of appetite. No changes in bowel habits, arthralgias/myalgias or any other complaint were present. He denied any travel abroad in the past year or any exposures that may determine etiology of the eosinophilia.

\section{Le rôle d'un traitement empirique à l'albendazole contre l'hyperéosinophilie idiopathique : une série de cas}

L'hyperéosinophilie est généralement définie comme une numération d'éosinophiles sanguins supérieure à $1500 / \mu \mathrm{L}$. Diverses affections s'y associent. Le présent rapport en décrit trois cas, dans lesquels des antécédents approfondis, un examen physique et des examens de laboratoire et d'imagerie n'ont pas décelé d'anomalies. L'albendazole a été administré de manière empirique dans les trois cas, et la numération d'éosinophiles s'est entièrement normalisée. Il faudrait envisager un traitement empirique à l'albendazole chez les patients présentant une hyperéosinophilie.

He was not taking any medications and had no medical history of disease. Complete blood count was performed adjacent to his clinical complaints. Leukocytosis of $23,000 / \mu \mathrm{L}$ with eosinophil count of $9430 / \mu \mathrm{L}$ was observed, but platelet and hemoglobin levels were normal. No blastocysts were found in the peripheral blood. Repeated stool samples for parasites and ova were negative. Chest $\mathrm{x}$-rays and electrocardiography were normal. The patient underwent a computed tomography (CT) scan of the chest, abdomen and pelvis, with no pathology found, except for a left inguinal hernia. A skin biopsy from his right leg revealed hyperkeratotic skin with mild angiocentric inflammatory changes, and no signs of vasculitis or malignancy. His bone marrow biopsy revealed normocellular bone marrow with increased number of eosinophils, which may be compatible with reactive changes. BCR$\mathrm{ABL}$ and Janus kinase-2 mutations were negative, as were tests for FIP1L1/PDG-FRA and FIP1L1/PDG-FRB mutations, ruling out myeloproliferative variant HES (M-HES). The patient was offered an empirical trial of albendazole ( $400 \mathrm{mg}$ twice a day for three days). The patient experienced complete resolution of hypereosinophilia following one week of albendazole treatment. There was no evidence of recurrence at the 10-month follow up visit.

\section{CASE 2}

A healthy, 23-year-old nonsmoker was admitted to the internal medicine department with abdominal pain and a three-week history of intermittent watery diarrhea. He did not experience vomiting, fever or night sweats, but complained of dyspareunia and approximately $2 \mathrm{~kg}$ of weight loss. The patient was on vacation in South America one month before admission. His physical examination appeared normal. Complete blood count revealed leukocytosis of $27,000 / \mu \mathrm{L}$ with eosino-

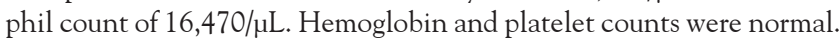
Blood chemistry and urinalysis were normal as well. Serology tests for HIV, hepatitis B and hepatitis C virus were negative. Stool cultures for bacteria and for the presence of Clostridium difficile were also negative.

${ }^{1}$ Department of Internal Medicine "E", Western Galilee Hospital, Nahariya; ${ }^{2}$ Department of Family Medicine, Clalit Health Services, Haifa and

Western Galilee district, Rapaport Faculty of Medicine, Technion, Haifa; ${ }^{3}$ Faculty of Medicine, Bar Ilan University, Zefat, Israel

Correspondence: Dr Faris Nassar, Western Galilee Hospital, Nahariya, Israel, POB 11, Naharia, Israel.

Telephone 972-4-9107451,972-50-7887619, fax 972-4-9107451, e-mail faris.nassar@naharia.health.gov.il 
Stool samples collected for three consecutive mornings, which were tested for parasites using wet mount examinations and concentration procedures, found no signs of helminthic infection. CT scans of the chest and abdomen exhibited increased wall thickness of the large intestine; however, gastroscopy and colonoscopy revealed normal mucosa appearance with no eosinophilic infiltration or parasites on biopsies. Flow cytometry revealed eosinophilia without any signs of malignancy. Bone marrow biopsy found an increased number of myeloid eosinophils with no signs of myeloproliferative disorder. Empirical therapy with albendazole (400 mg twice per day for three days) was initiated, followed by a decrease in eosinophil count in consecutive blood tests. One month after discharge eosinophil levels were normal.

\section{CASE 3}

A 32-year-old man, with a history of epilepsy for several years and with no history of travel or known exposure to helminths, was hospitalized in the internal medicine department after a short episode of unconsciousness without convulsions. Two days before his admission, he experienced right abdominal pain, without any fever or vomiting. His blood count revealed marked leukocytosis of $40,000 / \mu \mathrm{L}$ with eosinophil count of $26,000 / \mu \mathrm{L}$. Hemoglobin and platelet counts were normal. He had normal blood chemistry and no immature cells on blood smears. Flow cytometry and bone marrow biopsy revealed no sign of malignancy. BCR-ABL and Janus kinase-2 mutations were negative, as well as FIP1L1/PDG-FRA and FIP1L1/PDG-FRB mutations for detection of M-HES. CT scans of his chest and abdomen were normal. Serology for amoeba and strongyloides was negative. Gastroscopy with mucosal biopsy displayed signs of gastritis and Helicobacter pylori infection, but no signs of eosinophilic infiltration. Empirical therapy with albendazole (400 mg twice per day for three days) was initiated, followed by a rapid decline in eosinophil count.

\section{DISCUSSION}

Normal eosinophil count is usually approximately $400 / \mu \mathrm{L}$ (range $100 / \mu \mathrm{L}$ to $600 / \mu \mathrm{L})$. When elevated, eosinophil counts are arbitrarily classified as

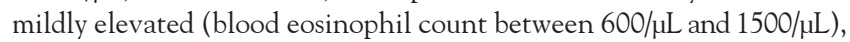
moderately elevated $(1500 / \mu \mathrm{L}$ to $5000 / \mu \mathrm{L})$ and severely elevated $(>5000 / \mu \mathrm{L})$. The finding of hypereosinophilia in any patient warrants clinical attention, especially when no apparent cause is found and counts are moderately or severely elevated.

Hypereosinophilia is most often divided into primary, secondary and idiopathic $(1,2)$. Secondary causes are the most commonly encountered, including helminthic infection, allergic disorders, medications, toxins, autoimmune diseases and endocrine disorders such as Addison disease. Rarely, neoplastic diseases, including varied adenocarcinomas, some forms of Hodgkin disease, T cell lymphoma and mastocytosis may be associated with paraneoplastic eosinophilia. Clues from the patient's history and physical examination can often guide toward a diagnosis. Primary hypereosinophilia is a clonal expansion of eosinophils, occurring in the context of hematological malignancy, such as acute leukemias and chronic myeloid disorders. HES' are a group of clinical disorders characterized by hypeosinophilia, accompanied by apparent organ damage/dysfunction, unexplained by secondary causes. Although once considered idiopathic, currently with modern diagnostic and management facilities, and recent advances in pathogenic mechanisms, criteria for HES' are being revised $(3,4)$.

A practical approach to managing a patient with hypereosinophilia has been discussed in the literature $(1,2)$. Diagnosis of a patient with hypereosinophilia usually includes a thorough history (including drug history and travel history) and physical examination, complete blood counts and blood smears, routine blood chemistry and several other laboratory examinations based on clinical suspicion. These include bone marrow aspirate and biopsy, karyotype and cytogenetics, parasite serology and stool samples for parasites, immunoglobulin E level, antineutrophil cytoplasmic antibody, troponin and vitamin $B_{12}$ levels, electrocardiogram and echocardiography, pulmonary function tests, and CT scans of the chest and abdomen. HES should be sought when previous tests have failed to detect the underlying cause. Tests for FIP1L1/PDG-FRA and FIP1L1/PDG-FRB mutations are currently recommended for the diagnosis of newly recognized M-HES (3). When no abnormality is found, 'wait-and-see' is the common recommended approach.

In the present report, we described three cases of hypereosinophilia in which a thorough history (including travel and drug history), physical examination and laboratory, as well as imaging investigations were unable to detect any abnormality. Although our patient in case 2 had recently traveled to South America, repeated stool cultures tested for parasites were completely normal. Our investigation included the most recently recommended tests for detection of M-HES. However, helminthic serologies and polymerase chain reaction testing for helminth parasites were not performed at our hospital. All three cases were treated empirically with $400 \mathrm{mg}$ of albendazole twice per day for three days, with complete normalization of eosinophil count thereafter.

Because albendazole has been reported to be effective in cases of idiopathic hypereosinophilia in tropical countries and countries in the Far East (where helminthic infection is more common) (5), our report demonstrated its efficacy in cases in the Western world. Therefore, we strongly suggest taking into consideration an empirical treatment using albendazole in cases in which thorough evaluation of hypereosinophilia has failed to detect the underlying cause.

DISCLOSURES: The authors have no financial relationships or conflicts of interest to declare.

\section{REFERENCES}

1. Roufosse F, Weller PF. Practical approach to the patient with hypereosinophilia. J Allergy Clin Immunol 2010;126:39-44.

2. Ang AL, Wong RX, Zhuang QY, Linn YC. Natural history of severe eosinophilia with uncertain aetiology and proposals on a practical approach to its management. Intern Med J 2012;42:928-33.

3. Simon HU, Rothenberg ME, Bochner BS, et al. Refining the definition of hypereosinophilic syndrome. J Allergy Clin Immunol 2010;12:45-9.

4. Klion A. Hypereosinophilic syndrome: Current approach to diagnosis and treatment. Annu Rev Med 2009;60:293-306.

5. Insiripong S, Siriyakorn N. Treatment of eosinophilia with albendazole. Southeast Asian J Trop Med Public Health 2008;39:517-20. 


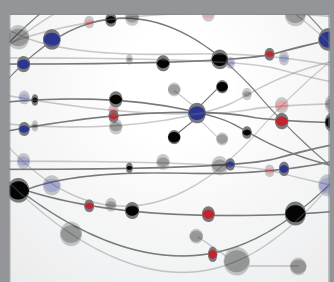

The Scientific World Journal
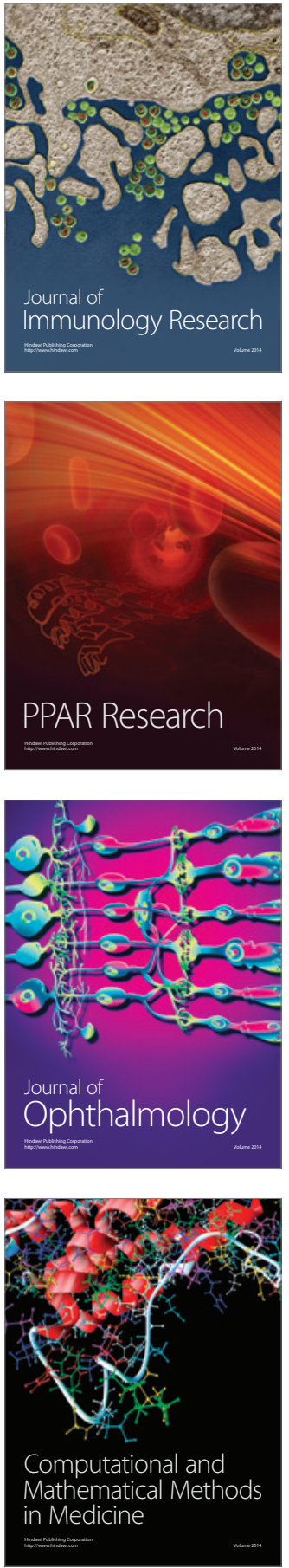

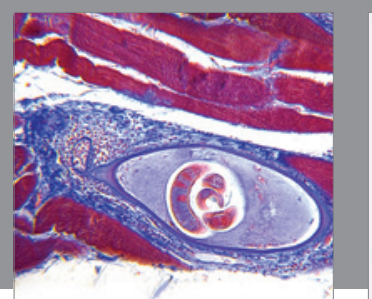

Gastroenterology Research and Practice

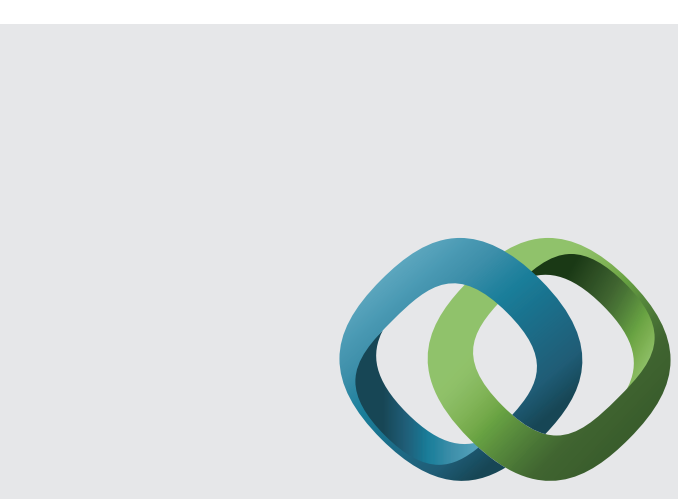

\section{Hindawi}

Submit your manuscripts at

http://www.hindawi.com
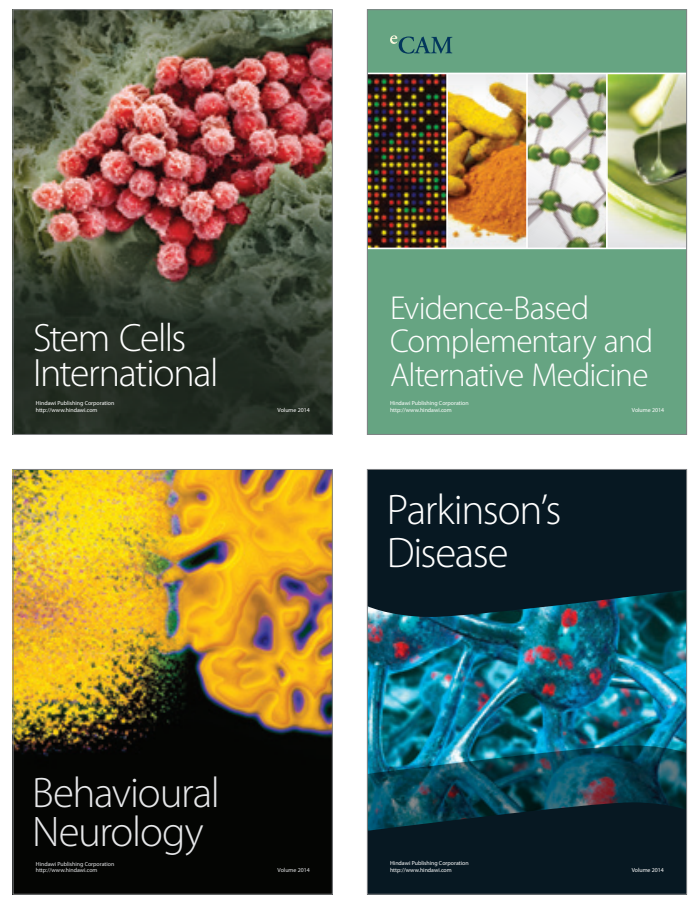
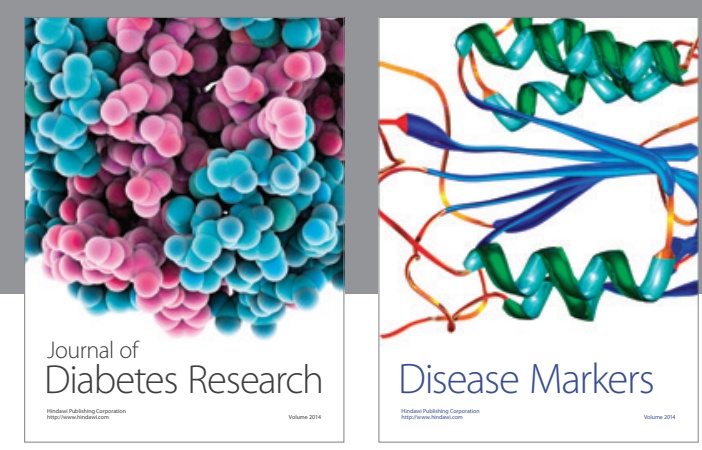

Disease Markers
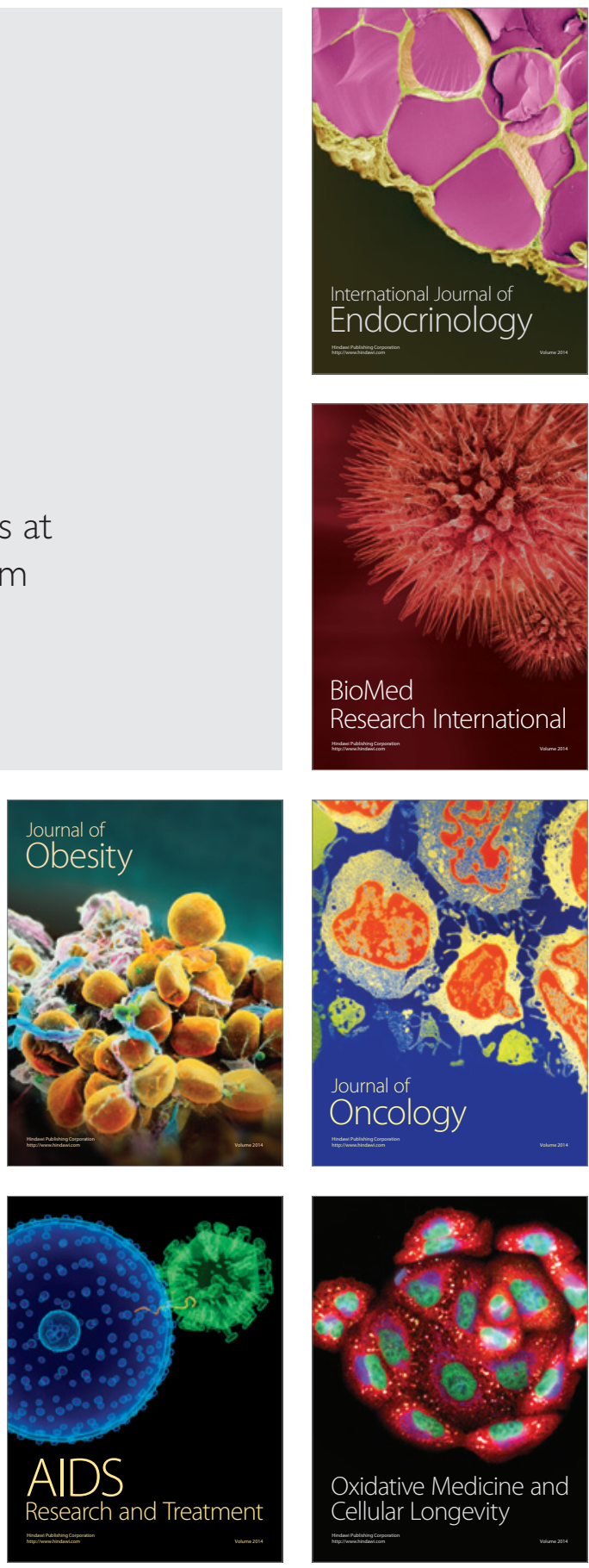